\title{
A Laboratory Technique for Evaluating Marine Splash Zone Corrosion
}

\author{
Xiang $\mathrm{Li}^{1, \mathrm{a}}$ and Stuart I. Bailey ${ }^{1, \mathrm{~b}}$ \\ ${ }^{1}$ Corrosion Centre for Education, Research and Technology, Department of Chemistry, Curtin University, \\ GPO Box U1987, Perth, WA, 6845, Australia
}

${ }^{a}$ xiang.li14@postgrad.curtin.edu.au, bs.bailey@curtin.edu.au (corresponding author)

\section{Keywords: Corrosion, Marine Splash Zone, Corrosion Resistant Alloys, Seawater}

\begin{abstract}
An electrochemical cell and environmental chamber were developed to study corrosion in a simulated splash zone. The designs of the dual chambers and the mounting of the three electrodes in the cell resulted in an excellent simulation of the major parameters and conditions in the marine splash zone. Excellent data acquisition and corrosion observations were possible, thereby, leading to a successful laboratory technique for research on corrosion in the marine splash zone. Electrochemical corrosion measurements correlated with the progression of changes during the wet - dry cycles, and the corrosion behavior was verified by microscopic observation.
\end{abstract}

\section{Introduction}

Renewable energy is increasingly produced from maritime locations. Tidal and wave power generation are inextricably intertwined with the marine environment. Wind turbines are often located offshore [1], primarily to avoid intrusion on terrestrial locations. Additionally, nonrenewable oil and gas production is also tending more and more to offshore locations [2-4]. Construction of massive infrastructure in offshore environments necessitates the use of appropriate corrosion resistant materials such as "stainless steels". A proper understanding of the corrosion properties of these materials of construction is vital to allow facilities to be designed with sufficient lifetime to provide the required service and avoid any potential for environmental impact which may arise from premature failure in these maritime locations.

An area which has received less attention is the "splash zone". This is the area above the low water mark, where waves, wind and tides cause periodic splashing with seawater. After a "wet" period, the seawater will undergo concentration by evaporation of water, primarily due to solar heating, and also with a likely contribution from the wind and complex interaction with the relative humidity. This is a complex environment, susceptible to corrosion, and it is difficult to make corrosion predictions due to the continual change in parameters such as temperature and dissolved salt concentration, which are key determinants of the corrosion behavior. In this paper we report a method developed to study splash zone corrosion in the laboratory, and results are given to illustrate the capabilities of the apparatus developed.

\section{Experimental}

In the conventional electrochemical corrosion test, the three electrode system $[5,6]$ is normally set in the bulk solution in a condition of full immersion. However, in the evaluation of marine splash zone corrosion, the surface of the specimen (electrodes) might only be covered by a certain volume electrolyte with a tiny thickness, instead of ample bulk solution, and it may be exposed to the air in the dry stage. Also, the cyclic wet-dry exposure condition caused by sunlight and evaporation in the splash zone will decrease the solution thickness, resulting in a higher salt concentration or crystal precipitation, which makes the solution conditions and corrosion phenomenon in the splash zone environment significantly different and more complicated than that in a bulk solution condition. Therefore, conventional corrosion test methods will not perform well. Decreased stability of the electrodes and more errors or failures in measurements will result when attempting to utilize the fully immersed three electrodes in a corrosion test for the splash zone. Hence, in order to overcome 
these problems and enhance the performance of electrodes, a new three electrode system was designed to operate beneath the bottom of the thin residual electrolyte layer by embedding electrodes into the epoxy resin base of the test cell. In addition, a dual chamber system was designed to control the ambient temperature and in particular the relative humidity.

Preparation of working electrode and counter electrode. The specimen (working electrode) was prepared as square steel coupons of $10 \mathrm{~mm}$ by $10 \mathrm{~mm}$ and $5 \mathrm{~mm}$ thickness. Before mounting into the epoxy resin, specimen underwent electrochemical coating of an organic resin, which was cured at $150^{\circ} \mathrm{C}$ for at least 1 hour, or set in the oven at $60^{\circ} \mathrm{C}$ for overnight. This coating procedure was vital to ensure that the occurrence of crevice corrosion could be essentially avoided at the four sides of the specimen when embedded into the epoxy resin. Meanwhile, the conventional platinum mesh counter electrode was replaced by a hastelloy rod. The hastelloy rod was mounted at $3 \mathrm{~mm}$ distance together with the specimen in the same epoxy resin.

Preparation of reference electrode. In the wet-dry exposure condition and the limited test area, the conventional double junction $\mathrm{Ag} / \mathrm{AgCl}_{\text {(satd) }}, \mathrm{KCl}_{\text {(satd) }}$ reference electrode was not suitable for direct measurement in the solution. Additionally, the traditional method of making contact of the solution with the reference electrode using a Luggin capillary was also unsuited due to its long length, thin bore, brittle and inflexible tip, which would induce high impedance to the test. In particular, once a bubble formed in the solution (which happens readily as the thickness of the surface electrolyte decreases), significant error would be introduced. As a result, two different reference electrodes were designed and tested. The first one was involved the combination of a selfmade salt bridge and an $\mathrm{Ag} / \mathrm{AgCl}$ reference electrode (Fig.1 left). The salt bridge was made of bent glass tubing and a cut plastic nozzle. The plastic nozzle and glass tubing both have a wider width than the bore of the Luggin capillary. Moreover, the plastic tip is flexible and easily cut into a flat surface, which could contribute more connectivity and less bubble generation. $3 \mathrm{M} \mathrm{KCl}$ solution + $2 \%$ agar gel were thoroughly mixed at boiling temperature and filled into the salt bridges without gaps or bubbles, while the mixture was approximately $80 \sim 90{ }^{\circ} \mathrm{C}$. All the salt bridges produced were stored in the $3 \mathrm{M} \mathrm{KCl}$ solution before starting experiments. The solution in the test tube holding the salt bridge and $\mathrm{Ag} / \mathrm{AgCl}$ reference electrode was the same as that in the chamber for the corrosion test.

In the second design, a hastelloy rod was embedded into the epoxy resin as a reference electrode, schematically shown in Fig.1 (right). The hastelloy is more stable and reliable [7] than the salt bridge, particularly when the conditions in the simulated splash zone became hot and dry. For many applications the stability of the relative potential between the working and reference electrodes is more important than the absolute potential value of the reference electrode, especially when it undergoes large deviations or fluctuations. Some electrochemical test techniques such as Electrochemical Impedance Spectroscopy [8,9], EIS, only investigate the impedance response from the equilibrium system by adding a tiny AC excitation signal, which is actually independent of the corrosion potential (or OCP value) of the specimen, versus the reference electrode. Therefore, the second design was acceptable and its usefulness was proved by further tests.
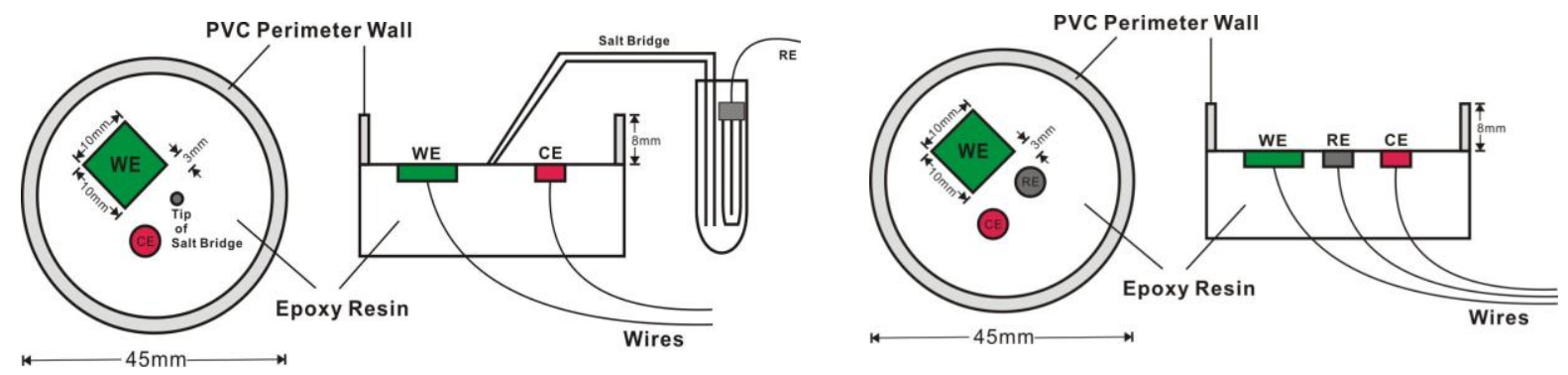

Fig.1 Schematic top and transverse cross-sectional views of three electrode cells employing salt bridge and Ag/AgCl reference electrode (left) and hastelloy reference electrode (right), respectively. 
Electrochemical chamber. The electrochemical chamber was based on a modified 6 L capacity acrylic container with dimensions of $25 \mathrm{~cm}(\mathrm{~L})$ x $20 \mathrm{~cm}(\mathrm{~W}) \times 14 \mathrm{~cm}(\mathrm{H})$, as shown in Fig. 2.
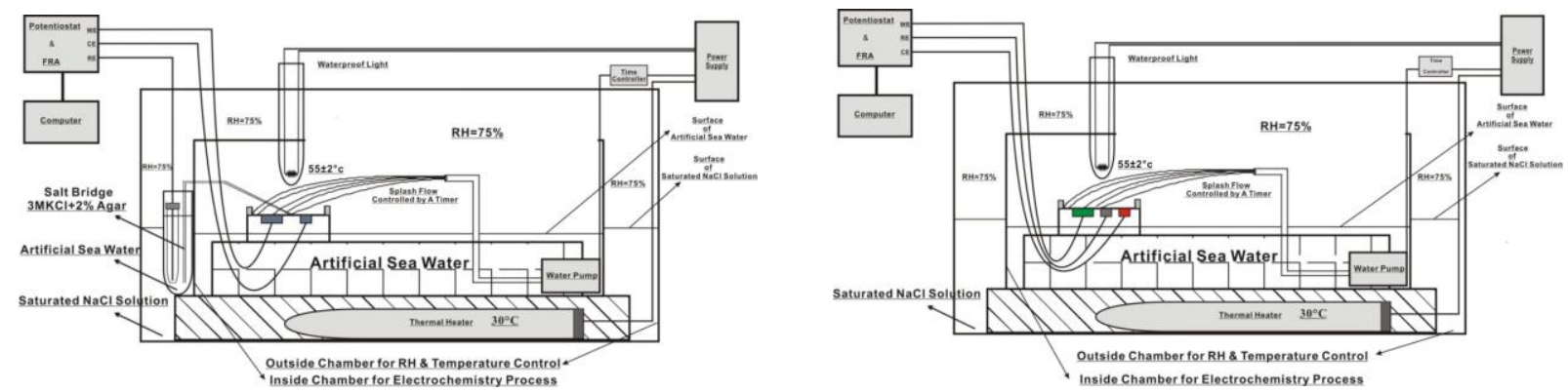

Fig.2 Dual chamber system and electrochemical cells connected with salt bridge and the $\mathrm{Ag} / \mathrm{AgCl}$ reference electrode (left) and hastelloy reference electrode (right), respectively.

A rectangular plastic rack holder was set on the bottom to hold the epoxy-mounted test sample steady and horizontally above the surface of the artificial sea water solution. A nozzle, connected to a water pump, was set on the opposite side of the rack holder to splash the solution onto the cell in a cyclic flow operation. The water pump was under the control of a timer. The PVC perimeter wall could maintain a constant volume of solution before each wet-dry exposure test. A water proof incandescent bulb light with power of $100 \mathrm{~W}$ was set above the sample surface at a height of $3.5 \mathrm{~cm}$ to simulate the surface temperature of $55 \pm 2^{\circ} \mathrm{C}$ which is common on a marine structure when the atmospheric temperature reaches $35-37^{\circ} \mathrm{C}$. When the solution on the sample surface had completely dried and before no more signal could be detected by the electrochemical instrument, the water pump controlled by the timer would automatically splash new solution into the PVC wall to start a new wet-dry exposure cycle. The entire electrochemical chamber was set in another sealed plastic container with a larger dimension. Saturated $\mathrm{NaCl}$ solution $(15 \mathrm{wt} \%$ over dose) was placed into this plastic container to keep the $\mathrm{RH}$ at near $75 \%$. The solution temperature was controlled by the thermal heater at $30^{\circ} \mathrm{C}$.

\section{Results and discussion}

In order to test the performance of the new design, the pitting corrosion behaviours of stainless steel (SS) 304 was investigated in a cyclical sequence (shown as Fig.3) by electrochemical techniques, such as open circuit potential (OCP) measurement and electrochemical impedance spectroscopy (EIS) measurement. An infinite focus microscope (IFM) was used to observe the corroded sample surface.

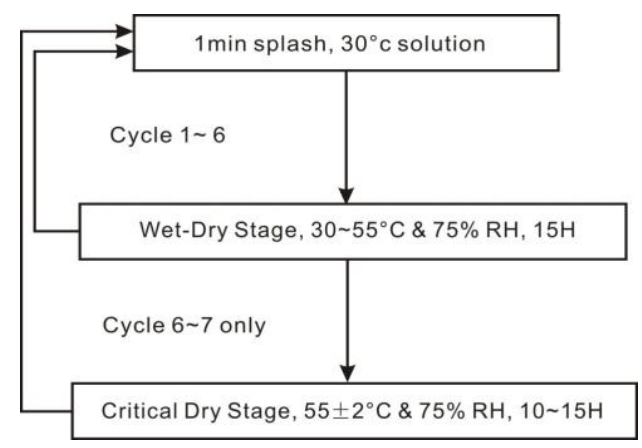

Fig. 3 the cyclic sequence of splash - evaporation cycles with extended critical dry stages.

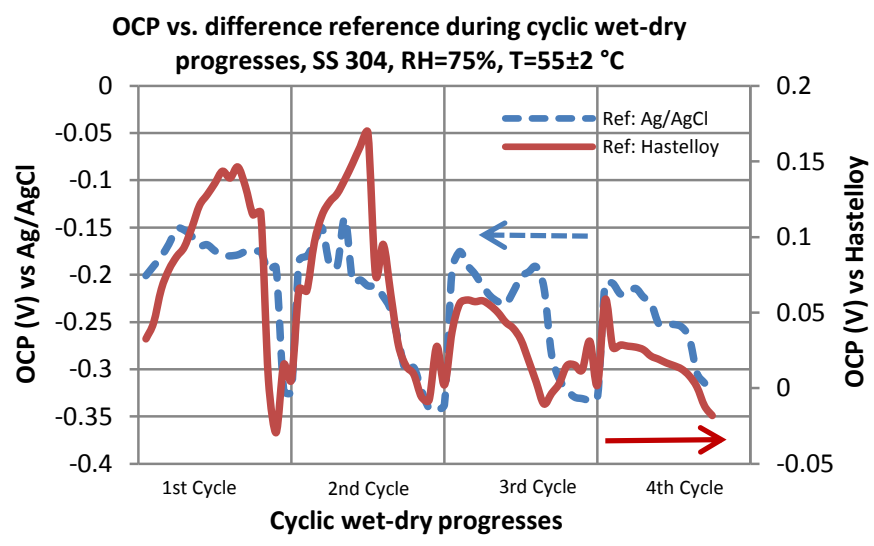

Fig.4 OCP values of SS 304 during four continuous wet-dry exposure cycles. 
Fig.4 shows the fluctuation of OCP values of SS 304 with different reference electrodes. Measurements were both carried out in the cyclic wet-dry chamber with the same test parameters. The blue/dashed line presents the OCP of type $304 \mathrm{SS}$ with saturated $\mathrm{Ag} / \mathrm{AgCl}$ reference electrode, while the red/solid line shows that using a hastelloy reference electrode. It is observable that both curves indicate the same regularity of the pitting corrosion performance with type 304 over the test periods. The OCP always dropped down in the latter stage of each cyclic period. This obvious shift shows that the protectivepassive film on the surface of the corroding stainless steel broke down and pitting corrosion occurred. The times for initiation and propagation of pitting corrosion were similar in each cycle, which suggests that the increasing chloride concentration caused by the evaporation played a major role in pits' formation. However, after each splash, a rapid positive shift could be observed in each cycle, which means the passivity returned again after the passive film had broken down at the dry stage in the preceding cycle. It is suggested that the splashing would flush away the surface precipitation and pit debris, and expose the pit zone with large surface to the oxygen dissolved in fresh splashing solution, which would enhance the re-passivation of the broken surface film and lead to a meta-stable stage of pitting corrosion. After that, the chloride concentration increased again in the next cycle and induced new penetration, resulting in passive film breakdown once again and propagation of pit growth, in accordance with the next cycle of a significant decline of OCP.

Fig.5 \& Fig. 6 below show the Bode plot from EIS measurement for SS 304 in 1st wet-dry cycle and $6^{\text {th }}$ wet-dry cycle, respectively. From curve \#1 to \#15 in Fig.5, the phase becomse more negative (black dashed circle zone), which means the capacitance (or complex impedance) of the sample surface was increasing [10] at the initial stage. Afterwards, the time constant peak and the phase at both high frequency (HF) and Low Frequency (LF) began to increase (downwards dashed arrow), indicating not only the breakdown of the oxide film formed at the initial immersion stage, but also the decrease of the capacitance at HF, which probably indicates further damage on the original passive film and the alteration of the properties of the thin electrolyte.

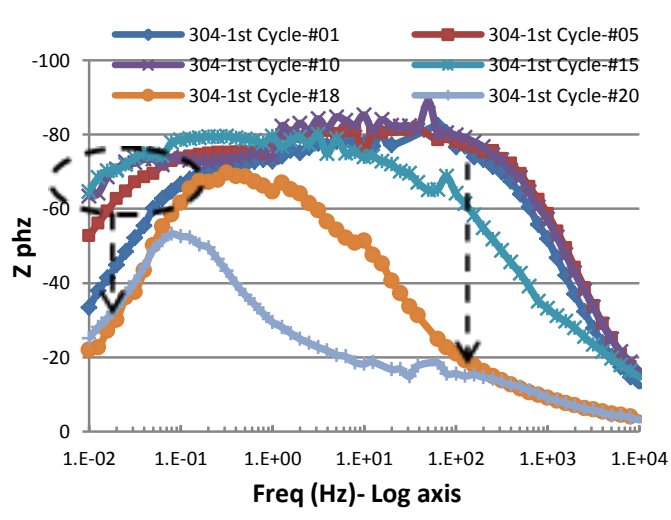

Fig.5 EIS Bode plot $1^{\text {st }}$ Cycle, SS 304

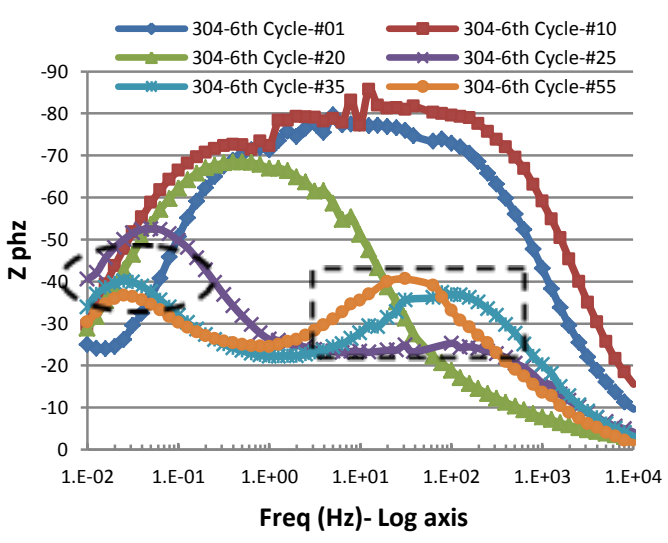

Fig.6 EIS Bode plot $6^{\text {th }}$ Cycle, SS 304

In Fig.6, with respect to the $1^{\text {st }}$ cycle, curves \#05, \#15 and \#18 were replaced by \#25, \#35, and \#55 because of not only the similarity of successive results, but also the insight provided for investigation of the critical dryness stage. In comparison with curves at LF in Fig.5, curves \#01 \#25 in the dashed circle all show more positive phase values, indicative of the more aggressive corrosion condition of $6^{\text {th }}$ cycle, lowering the impedance of the oxide film. Moreover, when the time constant peak at LF dropped, a new time constant peak formed at HF and gradually dominated the circuit (dashed rectangle). This phenomenon can be explained by the electrochemical pitting corrosion process with a high rate generating more corrosion products, which form a capacitive thick film on the pit surface and result in the increase of impedance. 
This proposition was proved by the observation of the noble shift of the OCP curves in Fig.7 due to the increase of the mixed potential [11]. The IFM image of Fig.9 shows morphological evidence of a compacted corrosion product layer

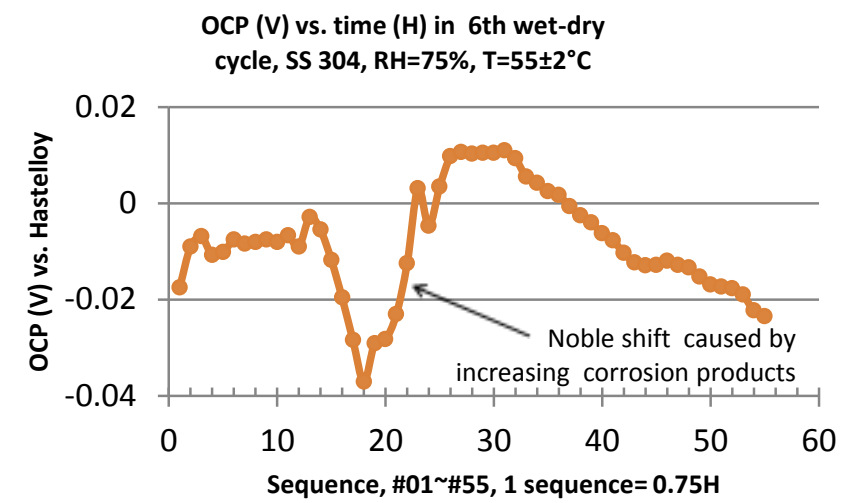

Fig.7 OCP of wet-dry stage $(0 \sim 15 H)+$ critical dry stage

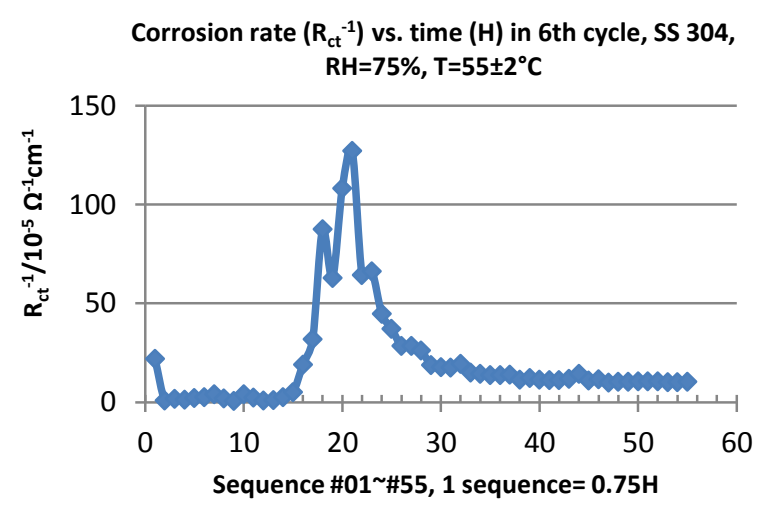

Fig.8 corrosion rate converted from EIS fit data

Fig.7 and Fig.8 both indicate an important phenomenon, which is that the dryness indicated by conventional investigation methods is not real dryness. The data acquisition after approximately 15 hours (sequence \#20) in the $6^{\text {th }}$ cycle using different measurements provided really good evidence to support this conclusion. In addition, it is well proved that the newly designed cell performed reliably and achieved perfect data acquisition under splash zone conditions. The IFM image of Fig.10 shows morphological evidence of trapped solution in the precipitated cystal, which is probably caused by the equilibrium between the solution evaporation and salt deliquescence at particular temperature and RH [12].

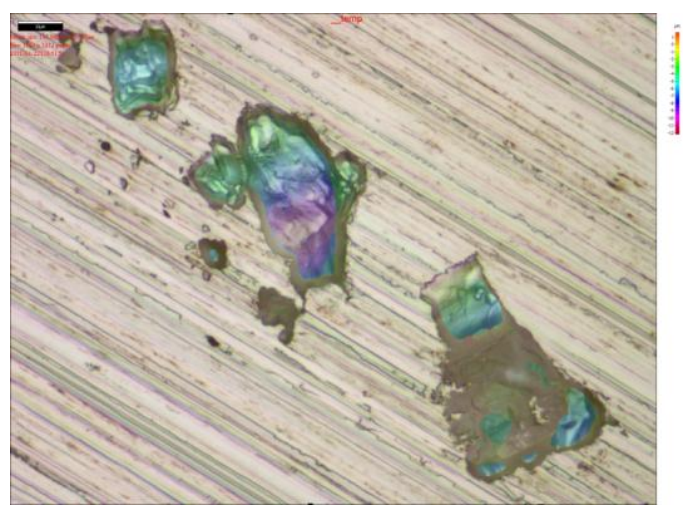

Fig.9 pits on surface of type 304,covered by passive corrosion product layer, forming $2^{\text {nd }}$ time peak at HF in EIS and noble shift in OCP.

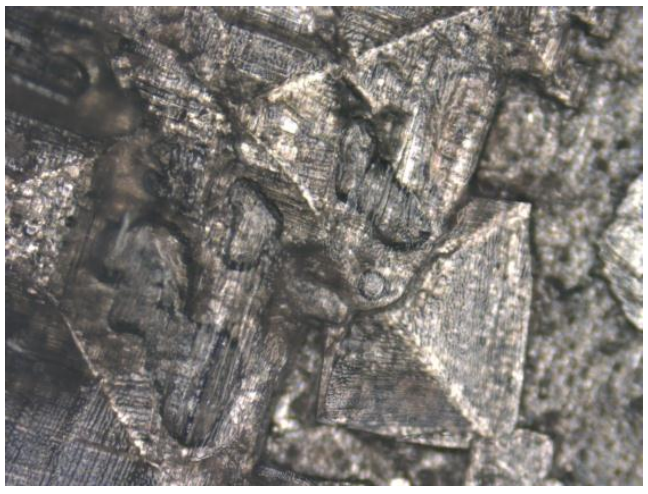

Fig.10 trapped solution by the precipitated cystal, keeping mositure between the sample surface and RE, resulting in long term conduction and corrosion at critical dry stage.

\section{Conclusions}

Apparatus was developed to allow corrosion measurement in an environment simulating the marine splash zone:

- Electrochemical measurements with this apparatus demonstrate the changing corrosion behavior as the environment changes during a wet - dry cycle.

- Electrochemical observations using the cell and chamber can be correlated with microscopic observations of the corroded steel surface.

- Sequential passive film breakdown, and re-passivation were shown to occur as the steel progressed toward dryness, and was then wet again. 


\section{Acknowledgements}

We acknowledge the support of the National Science Foundation of China No.40876048 and ChinaAustralia Special Fund for S\&T Cooperation No.41011120050.

\section{References}

[1] J. Twidell and G. Gaudosi, Offshore Wind Power, Multi-Science Publishing Company, Brentwood, 2009.

[2] .W. He, G. Jacobsen, T. Anderson, F. Olsen, T.D. Hanson, M. Korpås, T. Toftevaag, J. Eek, K. Uhlen and E. Johansson, The Potential of Integrating Wind Power with Offshore Oil and Gas Platforms, Wind Engineering, 34 (2010) 125-137.

[3] D. A. John, B. J. Kinsella, S. I. Bailey and R. De Marco, Flow Dependence of Carbon Dioxide Corrosion Using Short Electrodes by Jet Impingement, Corrosion - NACE, 65, 2009, 771 - 777.

[4] Y.-J. Tan, S. Bailey and B. Kinsella, Mapping non-uniform corrosion using the wire beam electrode method. I. Multi-phase carbon dioxide corrosion, Corrosion Science, 43 (2001) 19051918.

[5] R. de Marco, S. Bailey, J. Zhong-Tao, J. Morton and R. Chester, An In-situ Chronoamperometry/Synchrotron Radiation Grazing Incidence X-ray Diffraction Study of the Electrochemical Oxidation of Pyrite in Chloride Media, Electrochemistry Communications, 8 (2006) 1661 - 1664.

[6] I.M. Ritchie, S. Bailey and R. Woods, The Metal - Solution Interface, Advances in Colloid and Interface Science, 80, 1999, 183-231.

[7] V.Jovancicevic et al, Inhibition of $\mathrm{CO} 2$ corrosion of mild steel by imidazolines and their precursors, Corrosion 98 (1998), NACE International, U.S.A., paper No.18.

[8] S. Bailey, Y. J. Tan and B. Kinsella, Use of Electrochemical Impedance Spectroscopy for the Study of CO2 Corrosion Protection by Batch Treatment Inhibitors, British Corrosion Journal, 32 (1997) 49-55.

[9] B. Kinsella, Y. J. Tan and S. Bailey, Studies of CO2 Corrosion Product Scales using Electrochemical Impedance Spectroscopy and Surface Characterisation Techniques, Corrosion - NACE, 54 (1998) 835-842.

[10]Z. Keresztes et al, Role of redox properties of biofilms in corrosion processes, Electrochimica Acta, 46 (2001) 3841-3849.

[11]R.G. Kelly et al, Electrochemical Techniques in Corrosion Science and Engineering, Marcel Dekker, Inc., New York, 2003, chapter 7.

[12]Y. Tsutsumi et al, Monitoring of Rusting of Steels in Marine Atmosphere Using Electrochemical Impedance Technique, Journal of the Electrochemical Society, 153 (2006) B278-B282. 